\title{
Carta al editor en relación con el artículo: Experiencia inicial en pancreatoduodenectomía laparoscópica en un hospital general de la Ciudad de México ${ }^{1}$
}

\author{
Letter to the editor regarding the article: Initial experience in laparoscopic \\ pancreatoduodenectomy at a general hospital in Mexico City
}

Gerardo Miranda-Dévora,* Alejandro E Padilla-Rosciano, * Alberto M León-Takahashi, * Gonzalo Montalvo-Esquivel, ${ }^{* *}$ Horacio N López-Basave*

Estimada editora:

C on el advenimiento de la cirugía laparoscópica, desde la década de los 80 se ha desarrollado una evolución impactante en el tratamiento de las enfermedades quirúrgicas; por ejemplo, en la actualidad es difícil concebir la cirugía de hiato esofágico para el tratamiento de la enfermedad por reflujo gastroesofágico (ERGE) de manera abierta. La colecistectomía y la apendicectomía probablemente sean los procedimientos que más frecuentemente se realizan en el mundo y ahora, en nuestros días, los casos que se tratan con cirugía abierta son muy seleccionados y la razón parece ser más del tipo económica y de disponibilidad de recursos, que una indicación médica.

Con el desarrollo de las habilidades técnicas y la accesibilidad de la información, día a día, lo que antes se pensaba que sólo existía una manera de realizarse encuentra una nueva forma de abordaje; incluso, con el alcance de las redes sociales, es posible ser testigo en vivo de los acontecimientos que se comparten ya sea por fotos, video o cualquier medio electrónico, dando en ocasiones aquella sensación muy sutil y tal vez poco razonada de... "si ellos pueden... nosotros podemos".

Para las enfermedades no malignas, cuyo tratamiento está basado en la cirugía, cuando se realiza el procedimiento de mínima invasión y el resultado no es el esperado para el padecimiento, independientemente de la razón o la etiología, en la mayoría de los casos se puede optar por una cirugía de "Redo" la cual consiste en la reintervención, desmantelamiento o restauración de la anatomía previamente modificada con el fin de corregir la falla de la cirugía y tener así una oportunidad más que resuelva la enfermedad, ${ }^{2}$ o bien cuando es necesario se realiza una cirugía para control de daños cuya prioridad, más que resolver la enfermedad, es salvar la vida del paciente, en muchas ocasiones una segunda intervención puede ser realizada nuevamente por laparoscopia, y en algunos casos se opta por la cirugía abierta.

En el caso de las enfermedades malignas específicamente en los tumores sólidos, la cirugía continúa siendo la piedra angular en el tratamiento, la finalidad de los procedimientos es llevar a cabo una resección completa de la zona afectada y dependiendo de la histología se deben incluir los relevos ganglionares de la región tratada.

Cada día la cirugía laparoscópica gana un lugar muy específico, especialmente en los estadios clínicos tempranos, como forma de abordaje terapéutico sin sacrificar el objetivo oncológico.

Para poder medir el beneficio de los procedimientos laparoscópicos en el tratamiento de cáncer es necesario llevar a cabo complejos estudios de investigación para comparar el 
tratamiento estándar con el tratamiento de mínima invasión; en muchas ocasiones se ven involucrados distintos tipos de especialistas e incluso se llevan a cabo de manera multicéntrica y una vez que se tiene evidencia tangible que demuestre la "no inferioridad" del tratamiento se publican recomendaciones en organismos nacionales o internacionales los cuales lo categorizan en algo que se denomina "nivel de evidencia" para que así, el prestador de servicio final tome la responsabilidad del procedimiento teniendo en cuenta sus capacidades, disponibilidad de recursos y experiencia. ${ }^{3}$

La elección del tipo de abordaje terapéutico con respecto al orden de los procedimientos (quimioterapia, radioterapia o cirugía), es una decisión individual para cada centro hospitalario que maneja pacientes con cáncer, no sólo depende del estadio de la enfermedad y el estado funcional del paciente, también los recursos o capacidad de la institución tienen un papel importante. La evaluación integral del paciente permite al cirujano tomar decisiones que tendrán impacto en la supervivencia global, periodo libre de enfermedad y recurrencia, por lo que se le debe de ofrecer el tratamiento que se tenga documentado ofrezca los mejores resultados. En la última década cada vez son más los centros hospitalarios que publican los resultados de la pancreatoduodenectomía con técnica abierta versus laparoscópica, se debe tener cautela con la interpretación de datos. El que un procedimiento sea factible técnicamente y los resultados a corto plazo sean similares a los de la terapia estándar nos obliga a hacer el máximo esfuerzo para al menos cumplir con lo mínimo indispensable que el procedimiento demanda, como por ejemplo la adecuada obtención de márgenes y cosecha ganglionar, aunque para esto se tenga que optar por la conversión del procedimiento para alcanzar resultados óptimos. ${ }^{4-6}$

En el escenario en que la terapia que se propone no es considerada terapia estándar, como la pancreatoduodenectomía laparoscópica, y se ofrece una vía de abordaje alternativa, siempre debe ser bajo un protocolo de investigación que cubra los requerimientos de control de calidad para el tipo de enfermedad que permita medir de forma objetiva los resultados de la terapéutica; ${ }^{7}$ no se debe olvidar que la resección de la enfermedad maligna es sólo un fragmento del tratamiento que de realizarse de manera satisfactoria impactará no sólo en los primeros treinta días del postoperatorio. ${ }^{8}$ De ahí la importancia de abrir canales de comunicación con las distintas instituciones que cuenten con los equipos multidisciplinarios para el manejo del paciente con cáncer y sumar esfuerzos para el manejo de esta enfermedad.

"El cirujano debe reconocer el valor verdadero de sus intervenciones, las cuales requieren una evaluación sistemática y exhaustiva de los resultados en los pacientes."

\section{REFERENCIAS}

1. Crisanto-Campos BA, Arce-Liévano E, Robles-Aviña JA, Cárdenas-Lailson LE, Trejo-Ávila ME, Moreno-Portillo M. Experiencia inicial en pancreatoduodenectomía laparoscópica en un hospital general de la Ciudad de México. Cir Gen. 2016; 38: 59-66.

2. Desai AA, Alemayehu H, Dalton BG, Gonzalez KW, Biggerstaff B, Holcomb GW 3rd, et al. Review of the experience with re-operation after laparoscopic Nissen fundoplication. J Laparoendosc Adv Surg Tech A. 2016; 26: 140-143.

3. Poonacha TK, Go RS. Level of scientific evidence underlying recommendations arising from the national comprehensive cancer network clinical practice guidelines. J Clin Oncol. 2011; 29: 186-191.

4. Zhang YH, Zhang CW, Hu ZM, Hong DF. Pancreatic cancer: open or minimally invasive surgery? World J Gastroenterol. 2016; 22: 7301-7310.

5. Merkow J, Paniccia A, Edil BH. Laparoscopic pancreaticoduodenectomy: a descriptive and comparative review. Chin J Cancer Res. 2015; 27: 368-375.

6. Liao CH, Wu YT, Liu YY, Wang SY, Kang SC, Yeh CN, et al. Systemic review of the feasibility and advantage of minimally invasive pancreaticoduodenectomy. World J Surg. 2016; 40: 1218-1225.

7. Verbeke CS, Leitch D, Menon KV, McMahon MJ, Guillou PJ, Anthoney A. Redefining the R1 resection in pancreatic cancer. Br J Surg. 2006; 93: 1232-1237.

8. Adam MA, Choudhury K, Dinan MA, Reed SD, Scheri RP, Blazer DG 3rd, et al. Minimally invasive versus open pancreaticoduodenectomy for cancer: practice patterns and short-term outcomes among 7,061 patients. Ann Surg. 2015; 262: 372-377.

9. Porter GA, Skibber JM. Outcomes research in surgical oncology. Ann Surg Oncol. 2000; 7: 367-375.

Correspondencia:

Gerardo Miranda Dévora

Instituto Nacional de Cancerología.

Av. San Fernando Núm. 22,

Colonia Sección XVI,

Del. Tlalpan, 14080, Ciudad de México, México. E-mail: gmirandad511@gmail.com 